\title{
Percepção de Risco da Cirurgia Bariátrica em Pacientes com Diferentes Comorbidades Associadas à Obesidade
}

\author{
Fabiana Brum Schakarowski ${ }^{1, *}$ \\ Orcid.org/0000-0003-0486-4909 \\ Alexandre Vontobel Padoin ${ }^{2}$ \\ Orcid.org/0000.0002.9754.4818 \\ Cláudio Corá Mottin ${ }^{2}$ \\ Orcid.org/0000-0002-1638-2248 \\ Elisa Kern de Castro ${ }^{1}$ \\ Orcid.org/0000-0002-1290-7561
}

\author{
${ }^{1}$ Universidade do Vale do Rio dos Sinos, São Leopoldo, RS, Brasil \\ ${ }^{2}$ Pontificia Universidade Católica do Rio Grande do Sul, Porto Alegre, RS, Brasil
}

\section{Resumo}

A etapa pré-operatória de cirúrgica bariátrica exige que paciente esteja informado sobre as comorbidades associadas à obesidade e dos riscos, benefícios e consequências da cirurgia. $\mathrm{O}$ estudo examinou a percepção de risco da cirurgia bariátrica em 128 pacientes, com diferentes comorbidades associadas (doenças cardíacas, diabetes mellitus tipo 2, hipertensão arterial, apneia do sono e dislipidemia) no pré-operatório de cirurgia bariátrica. A percepção de risco da cirurgia e das comorbidades associadas foram avaliadas através de um Questionário de Percepção de Risco. Os pacientes com diabetes mellitus tipo 2, hipertensão arterial e apneia do sono apresentaram avanço mais significativo, em relação as demais comorbidades, para a percepção de risco da cirurgia. Os resultados mostraram que as intervenções educativas expandem a percepção de risco da cirurgia, mas é independente o risco percebido entre as diferentes comorbidades dos pacientes obesos. $\mathrm{O}$ estudo aponta a possibilidade de identificar fatores preditivos do comportamento implícito relacionados com a percepção de risco para melhorar o resultado do tratamento a curto e longo prazo, nos pacientes cujas comorbidades são vistas como não oferecendo risco adicional à saúde.

Palavras-chave: Percepção de risco, obesidade, cirurgia bariátrica.

\section{Risk Perception of Bariatric Surgery in Patients with Different Obesity-Related Comorbidities}

\begin{abstract}
The preoperative stage of bariatric surgery requires that the patient be informed of the obesity-related comorbidities and the risks, benefits, and consequences of surgery. The study examined the preoperative
\end{abstract}

Endereço para correspondência: Universidade do Vale do Rio dos Sinos, Av. Unisinos, 950, Cristo Rei, São Leopoldo, RS, Brasil 93020-190. E-mail: fabwski@gmail.com, alexandre.padoin@pucrs.br, claudio.mottin@, pucrs.bre elisakc@unisinos.br 
risk perception of bariatric surgery in 128 patients with different comorbidities (heart disease, type 2 diabetes mellitus, hypertension, sleep apnea and dyslipidemia). Risk perception of surgery and associated comorbidities were evaluated using a risk perception questionnaire. Patients with diabetes mellitus type 2 , systemic hypertension, and sleep apnea were significantly more likely to perceive the risk of surgery compared to those with the other comorbidities. The results showed that educational interventions expand the risk perception of surgery, but independently of the perceived risk of the different comorbidities of obese patients. The study points to the possibility of identifying predictive factors of behavioral intention related to risk perception to improve short and long-term treatment outcomes in patients whose comorbidities are seen as posing no additional health risk.

Keywords: Risk perception, obesity, bariatric surgery.

\section{La Percepción del Riesgo de la Cirugía Bariátrica en Pacientes con Distintas Comorbilidades Asociadas con la Obesidad}

\section{Resumen}

El preoperatorio de cirugía bariátrica requiere que el paciente sea informado de las comorbilidades asociadas a la obesidad y los riesgos, beneficios y consecuencias de la cirugía. El estudio examinó la percepción de riesgo de la cirugía bariátrica en 128 pacientes con diferentes comorbilidades (enfermedades del corazón, diabetes mellitus tipo 2, hipertensión arterial, apnea del sueño y dislipidemia) en el preoperatorio de la cirugía bariátrica. La percepción de riesgo de la cirugía y las comorbilidades asociadas se evaluó mediante un cuestionario de percepción del riesgo. Los pacientes con diabetes mellitus tipo 2, hipertensión arterial y apnea del sueño tenían avance más significativo, en comparación con otras comorbilidades, a la percepción de riesgo de la cirugía. Los resultados mostraron que las intervenciones educativas ampliar la percepción del riesgo de la cirugía, pero es independiente de la percepción de riesgo entre las diferentes comorbilidades de los pacientes obesos. El estudio apunta a la posibilidad de identificar factores predictivos de comportamiento implícita relacionada con la percepción de riesgo para mejorar el resultado del tratamiento a corto y largo plazo en pacientes cuya comorbilidades son vistos como no proporcionar riesgo para la salud adicional.

Palavras clave: Percepción del riesgo, obesidad, cirúgia bariátrica.

A cirurgia bariátrica tem sido considerada eficaz para a perda de peso significativo, melhora das comorbidades e aumento da longevidade de pacientes obesos (Brzozowska, Sainsbury, Eisman, Baldock, \& Center, 2013; Sjöström et al., 2004). No entanto, não pode ser percebida apenas como uma ação técnica, sustentada num modelo ideal de saúde contemporâneo (Holtorf, Rinde, Rupprecht, Alder, \& Brixner, 2012). Desde o início do tratamento para a cirurgia bariátrica o paciente deve conhecer o diagnóstico da sua doença (obesidade) e julgar riscos, benefícios e consequências da cirurgia (Adams et al., 2007; Alvarez, Singh, \& Sinha, 2014; Kubik, Gill, Laffin, \& Karmali, 2013).
Sabe-se que comorbidades clínicas nem sempre são revertidas quando pacientes reganham peso em longo prazo e quando distúrbios psíquicos estabelecidos desde a infância precedem a cirurgia bariátrica (Ribeiro, Santos, \& Loureiro, 2011). Por isso, é importante que o paciente esteja ciente das mudanças comportamentais intrínsecas ao procedimento cirúrgico, que são determinantes para resultado sustentável do tratamento em longo prazo (Friedman, Applegate, \& Grant, 2007; Murguía et al., 2012). Expectativas irreais sobre a cirurgia bariátrica e seus riscos podem prejudicar mudanças necessárias no estilo de vida dos pacientes (Kubik et al., 2013; Murguía et al., 2012). Portanto, a cirurgia 
bariátrica deve ser empregada para complementar outras formas de tratamento da obesidade (Cohen, Schiavon, \& Torres, 2010; Flum et al., 2009).

As pessoas tendem a avaliar situações de risco baseadas, em grande parte, na probabilidade de consequências de doenças associadas (Shiloh, Wade, Roberts, Alford, \& Biesecker, 2013), que precipitam as complicações que podem ocorrer durante e após a cirurgia (Schauer et al., 2013; Wee et al., 2013; Wrzesinski et al., 2015). No entanto, o risco é um construto subjetivo cujas consequências de resultados podem diferir entre os indivíduos. De acordo com Slovic, Finucane, Peters e MacGregor (2004), o risco só pode ser mensurado dentro de um contexto, adquirindo significado em oposição a outros dados que interferem na sua construção. Os autores reconhecem que o risco e as respostas ao risco perpassam por valores do indivíduo, estabelecendo que não seja o risco real que afeta as decisões, mas sim a percepção de risco.

A percepção de risco, por sua vez, pode ser medida em termos de gravidade percebida da doença, da ocorrência do risco em si, ou pelo grau de preocupação e medo associados, exigindo um julgamento intelectual do indivíduo sobre a doença e seus fatores de risco (Sjöberg, 1998). Sendo assim, para melhor resultado do tratamento e reorganização positiva do estilo de vida, é necessário que o paciente receba todas as orientações antes da cirurgia, promovendo o juízo de riscos e engajamento de comportamentos preventivos à promoção de sua saúde (Kaly et al., 2008; Sjöström et al., 2007).

Pouco se sabe a respeito dessa percepção de risco em pacientes obesos, considerando as diferentes comorbidades associadas. Detectar as diferentes distorções na percepção de risco da cirurgia e das comorbidades associadas pode reforçar à equipe multidisciplinar apurar estratégias de enfrentamento com os pacientes cujas comorbidades são vistas como não oferecendo risco adicional ao curso da doença. Nesse contexto, o presente estudo objetivou examinar a percepção de risco no pré-operatório de cirurgia bariátrica em pacientes com diferentes comorbidades associadas à obesidade.

\section{Método}

Trata-se de estudo analítico, observacional e correlacional, com o propósito de examinar as associações entre as variáveis investigadas.

\section{Participantes}

A amostra foi constituída de 128 pacientes, candidatos à cirurgia de bypass gástrico num serviço terciário para tratamento da obesidade num hospital de Porto Alegre, Brasil, entre setembro de 2014 e janeiro de 2015. Os participantes eram maiores de 18 anos, alfabetizados, sem comprometimento intelectual ou limitação cognitiva.

\section{Instrumentos}

Questionário Sociodemográfico. Instrumento elaborado para o estudo, sendo os dados coletados a partir da revisão de prontuários da equipe médica: sexo, idade, situação conjugal, grau de escolaridade, situação laboral, índice de massa corporal (IMC), tempo de evolução da obesidade, tempo de espera para cirurgia e histórico de comorbidades.

Questionário de Percepção de Risco. Instrumento baseado no estudo de Prasad et al. (2014) e projetado para avaliar a percepção de risco da cirurgia e das comorbidades associadas (doenças cardíacas, diabetes mellitus tipo 2, hipertensão arterial, apneia do sono e dislipidemia). Através de uma escala Likert de 5 pontos $(1=$ sem risco, $2=$ risco baixo, $3=$ risco médio, $4=$ risco alto 5 = risco muito alto), os participantes avaliaram a percepção de risco das comorbidades através de duas perguntas: - "Antes de ingressar no programa de tratamento, quanto você se considerava em risco para as comorbidades associadas?" "Desde que está inserido no programa de tratamento, quanto você se considera em risco para as comorbidades associadas?". A percepção de risco da cirurgia foi avaliada sobre o quanto o paciente considerava um risco para sua saúde: fazer a cirurgia, não fazer a cirurgia e de morte pelas consequências da cirurgia. O questionário foi aplicado no ambulatório do Hospital, na véspera da cirurgia. Ainda não existe instrumento validado para avaliar o construto da Percepção de risco. Embora o uso de escalas padronizadas 
aumente a capacidade de reproduzir resultados, o questionário utilizado no presente estudo favoreceu avaliar como as diferentes comorbidades estão associadas com a percepção de risco da cirurgia.

\section{Procedimento de Coleta de Dados}

Após o tratamento pré-operatório com a equipe multidisciplinar e assistir as duas palestras obrigatórias, todos os pacientes convidados, que estavam prestes a fazer a cirurgia, aceitaram participar do estudo e assinaram o Termo de Consentimento Livre e Esclarecido. As palestras educativas são ministradas por especialistas do próprio Serviço, sendo realizadas em encontro mensal para cada especialidade. Estas palestras abrangem compreensão realista da doença e sustentam, dentre outros fatores, que a cirurgia bariátrica favorece a remissão de comorbidades associadas, mas, para eficácia do tratamento, é necessário autorregulação contínua sobre a doença e os padrões habituais inadequados, visando melhoria da qualidade de vida. Não há limite para assistir as palestras educativas, pois o paciente é sempre encorajado a expandir maior controle pessoal sobre sua doença, independente do tempo de espera pela cirurgia, minimizando os riscos da cirurgia e complicações pós-operatórias e melhor adesão ao tratamento.

\section{Procedimento de Análise de Dados}

Os dados quantitativos foram descritos por média e desvio padrão ou mediana e amplitude interquartil na quebra de pressupostos distribucionais. Dados categóricos foram apresentados por contagem e percentuais. A análise univariada envolveu a utilização do teste $t$ ou seu substituto não paramétrico para variáveis quantitativas e do teste de qui-quadrado ou exato de Fischer para dados categóricos. Adicionalmente, os dados relacionados à percepção de risco da cirurgia foram avaliados em um modelo de regressão logística ordinal, tomando como fator principal o sexo do paciente e ajustando para potenciais efeitos confundidores (escolaridade, idade $<45$ e $>45$, DM2 e hipertensão arterial). O nível de significância adotado foi de $5 \%$. Os dados foram analisados usando o programa estatístico Statis- tical Package for the Sciences (SPSS), versão 22.0 (SPSS Inc., Chicago, IL, USA).

\section{Procedimentos Éticos}

O estudo foi aprovado pelos Conselhos de Ética das Instituições envolvidas. Demais preceitos éticos e técnicos foram preconizados pela Resolução no 196/1996, do Conselho Nacional de Saúde (CNS).

\section{Resultados}

A amostra consistiu de 128 pacientes, 93 mulheres $(72,7 \%)$ e 35 homens $(27,3 \%)$, com idade média de 39,5 anos $(D P=11,0)$. A maioria dos pacientes tinha secundário completo (57,0\%), seguido do ensino superior $(31,2 \%)$. Com relação à situação conjugal, $68,8 \%$ afirmaram que eram casados. O tempo médio de espera para cirurgia bariátrica foi de 10,44 meses $(D P=7,7)$. O tempo de evolução da obesidade foi 16,2 anos $(D P=7,9)$. A maioria exercia atividade laboral (74,2\%). A média do Índice de massa corporal (IMC) dos participantes na véspera da cirurgia era de $47,00 \mathrm{~kg} / \mathrm{m} 2(D P=7,19)$. Entre as comorbidades, $3,1 \%$ dos pacientes tinham doenças cardíacas, $26,6 \%$ diabetes mellitus tipo 2, 63,3\% hipertensão arterial, 31,3\% apneia do sono e $66,4 \%$ dislipidemia. O detalhamento de dados sociodemográficos e clínicos pode ser visualizado na Tabela 1.

$\mathrm{Na}$ análise univariada, a percepção de risco para doenças cardíacas foi maior em homens do que em mulheres quando entraram no programa de tratamento $(\mathrm{OR}=0.5,95 \% \mathrm{CI}=0.2-0.9, p$ $=0,047)$. Os pacientes com maior IMC, na véspera da cirurgia, apresentaram maior percepção de risco para doenças cardíacas (OR 0.9, 95\% $\mathrm{CI}=0.9-1.0, p=0,029)$, diabetes mellitus tipo 2 (OR 0.9, 95\% CI $=0.9-1.0, p=0,001)$ e de morte pelas complicações da cirurgia (OR 0.9, 95\% $\mathrm{CI}=0.9-1.0, p=0,001)$. Além disso, na véspera da cirurgia, pacientes com doenças cardíacas relataram maior probabilidade de perceber os riscos de fazer a cirurgia (OR 1.5, 95\% CI=1.2-1.9, $p=0,001$ ) e de não fazer a cirurgia (OR 1.6, 95\% $\mathrm{CI}=1.2-2.1, p=0,001)$. Pacientes com diabetes mellitus tipo 2 , na véspera da cirurgia, também 
Tabela 1

Características Sociodemográficas e Clínicas dos 128 Pacientes

\begin{tabular}{lccc}
\hline \multicolumn{1}{c}{ Variáveis } & $\begin{array}{c}\text { Homens } \\
(n=35)\end{array}$ & $\begin{array}{c}\text { Mulheres } \\
(n=93)\end{array}$ & P value \\
\hline Idade & & & 0,675 \\
$<45$ & $25(71.4 \%)$ & $62(66.7 \%)$ & \\
$>45$ & $10(28.6 \%)$ & $31(33.3 \%)$ & \\
Com companheiro & $22(62.9 \%)$ & $66(71.0 \%)$ & 0,398 \\
Escolaridade & & & \\
Primário & $5(14.3 \%)$ & $10(10.8 \%)$ & \\
Secundário & $14(40.0 \%)$ & $59(63.4 \%)$ & \\
Superior & $16(45.7 \%)$ & $24(25.8 \%)$ & \\
IMC (kg/m $\left.{ }^{2}\right)$ & & & 0,110 \\
$\geq 35$ a $\leq 39.9$ & $1(2.9 \%)$ & $13(14.0 \%)$ & \\
$>40$ & $34(97.1 \%)$ & $80(86.0 \%)$ & \\
Tempo evolução da obesidade (anos) & $16.9 \pm 9.1$ & $16.0 \pm 7.5$ & 0,562 \\
Tempo espera para cirurgia bariátrica (meses) & $12.9 \pm 10.8$ & $9.5 \pm 5.9$ & 0,023 \\
Histórico de comorbidades & & & \\
Doenças cardíacas & $2(5.7 \%)$ & $2(2.2 \%)$ & 0,301 \\
Diabetes mellitus tipo 2 & $12(34.3 \%)$ & $22(23.7 \%)$ & 0,264 \\
Hipertensão arterial & $25(71.4 \%)$ & $56(60.2 \%)$ & 0,305 \\
Apneia do sono & $13(37.1 \%)$ & $27(29.0 \%)$ & 0,398 \\
Dislipidemia & $28(80.0 \%)$ & $57(61.3 \%)$ & 0,059 \\
\hline
\end{tabular}

Nota $. \mathrm{IMC}=$ índice de massa corporal.

perceberam maior risco de fazer a cirurgia (OR $1.4,95 \% \mathrm{CI}=1.2-1.8, p=0,001)$, de não fazer a cirurgia (OR 1.7, 95\% CI=1.3-2.3, $p=0,001$ ) e de morte pelas complicações da cirurgia (OR 1.6, $95 \% \mathrm{CI}=1.3-2.1, p=0,001)$. Para hipertensão arterial (OR 1.6, 95\% CI=1.2-2.2, $p=0,001)$ e dislipidemia (OR 1.5, 95\% $\mathrm{CI}=1.2-2.0, p=0,001)$ os pacientes tiveram maior percepção de risco para morte pelas complicações da cirurgia. Pacientes com apneia do sono (OR 1.6, 95\% CI=1.2-2.0, $p=0,001)$ tiveram maior percepção de risco de não fazer a cirurgia.

Os resultados ajustados da análise da Regressão Logística Ordinal revelaram que a percepção de risco da cirurgia, de fazer a cirurgia, de não fazer a cirurgia, e de morte pelas complicações da cirurgia foram muito similares entre os sexos. Após, o ajuste da análise multivariada, os homens apresentaram $60 \%$ maior percepção de risco de morte pelas complicações da cirurgia do que as mulheres. Os dados da análise são mostrados na Tabela 2. Não foram observadas diferenças significativas na análise para as demais multivariadas.

\section{Discussão}

Os resultados evidenciam que existe variação na percepção de risco da cirurgia e das comorbidades associadas após o atendimento multidisciplinar e das palestras educativas obrigatórias para efetivar a cirurgia bariátrica. Os pacientes homens buscam tratamento para obesidade com o quadro de comorbidades em estágio 
Tabela 2

Odds Ratio Não Ajustada e Ajustada por Sexo do Paciente

\begin{tabular}{lcccccc}
\hline & \multicolumn{3}{c}{ OR Não Ajustada } & OR ajustada \\
\cline { 2 - 7 } & OR* & $95 \%$ CI & P value & OR & $95 \%$ CI & P value \\
\hline Risco da cirurgia & 1.3 & $0.6-2.9$ & 0,512 & 1.3 & $0.6-3.0$ & 0,540 \\
Risco de fazer a cirurgia & 0.8 & $0.4-1.6$ & 0,593 & 0.9 & $0.4-1.9$ & 0,853 \\
Não fazer a cirurgia & 0.6 & $0.3-1.2$ & 0,137 & 0.5 & $0.2-1.3$ & 0,189 \\
Morte pelas complicações da cirurgia & 1.7 & $0.7-4.0$ & 0,202 & 1.6 & $0.7-3.8$ & 0,255 \\
\hline
\end{tabular}

Nota. $\mathrm{OR}=$ odds ratio.

mais avançado da doença, estando mais propensos em perceber o risco para as doenças cardíacas, quando comparados com as mulheres. Os resultados foram compatíveis com estudos anteriores, reforçando que os homens encontram-se metabolicamente menos saudáveis ao se submeterem a cirurgia (Chang et al., 2014; Lee et al., 2015). As mulheres buscam a cirurgia bariátrica com o andamento de comorbidades associadas em estágio menos avançado da doença, possivelmente, porque estão mais preocupadas com o padrão estético social (Pona, Heinberg, Lavery, Ben-Porath, \& Rish, 2016; Steffen et al., 2012), mesmo que não seja um critério de indicação para cirurgia.

No presente estudo, pacientes com diabetes mellitus tipo 2 mostraram altamente significativa a percepção de risco em não fazer a cirurgia, entre antes de entrar no programa de tratamento e a véspera da cirurgia. Esse aumento na percepção de risco de pacientes com diabetes mellitus tipo 2 pode ser explicado pelo fato dessa doença ter sintomas inicias silenciosos e, após as palestras educativas, pacientes ampliam seu entendimento sobre as graves consequências dessa doença, que pode levar a possíveis amputações, insuficiência renal e perda da visão. Outros estudos ressaltam a importância de intervenções educativas para promover o conhecimento da doença (Bonsaksen, Lerdal, \& Fagermoen, 2015; Breland, Fox, Horowitz, \& Leventhal, 2012; Jumean et al., 2012) onde o paciente ao se perceber em maior risco, pode desenvolver maior controle pessoal e modificar seu comportamento. Isso revela que, ao perceberem mais consequências da própria doença, os pacientes podem combater a intensi- dade e duração das comorbidades associadas à obesidade para amenizar os riscos da cirurgia.

Nossos resultados revelam ainda que os pacientes com hipertensão arterial, diabetes mellitus tipo 2 e apneia do sono, após receberem todas as informações sobre riscos e benefícios da cirurgia, tiveram avanço mais significativo de percepção de risco da cirurgia. Em contrapartida, na véspera da cirurgia, os pacientes com dislipidemia apresentaram redução na percepção de risco em fazer a cirurgia. Isso nos dá indício para compreender que o risco/benefício percebido da cirurgia bariátrica pode amparar a decisão desses pacientes, baseado no fato que o risco de morrer pelas comorbidades associadas pode ser maior do que o risco de fazer a cirurgia. Resultados semelhantes foram observados no estudo de Prasad et al. (2014), que concluiu que os pacientes submetidos a cirurgia bariátrica são mais propensos a perceber a obesidade como risco maior para a saúde do que a intervenção cirúrgica em si. Inversamente, pacientes em tratamento clínico subestimam o risco cardiovascular em longo prazo e superestimam o risco cirúrgico. É preciso que os pacientes se percebam como agentes ativos nesse processo, com percepção de risco preditiva associada com a cirurgia bariátrica e comorbidades relacionadas a obesidade, buscando motivação para aderir ao tratamento a curto e longo prazo e com sucesso da intervenção.

O presente estudo tem algumas limitações. Os pacientes estavam prestes a operar e tinham recebido informações pela equipe multidisciplinar, sobre todos os riscos e benefícios envolvidos na cirurgia. É possível que ao julgarem a cirurgia como um ganho maior, com expectati- 
va de "cura" da obesidade e de emagrecimento rápido, a preocupação com as comorbidades consideradas, talvez, menos insidiosas, fique atenuada. Nossos achados confirmam a complexidade da doença de obesidade e a importância dos aspectos psicológicos envolvidos, tanto para o agravamento do quadro, como para obtenção e manutenção de resultados duradouros do tratamento. Assim, sugere-se que futuras pesquisas investiguem aspectos envolvidos na identificação de fatores preditivos da intenção comportamental relacionados com a percepção de risco. Além disso, o questionário de Percepção de Risco poderá ser aprimorado e aplicado em diferentes tempos, antes da primeira avaliação clínica, imediatamente após a cirurgia e decorridos meses da cirurgia.

\section{Considerações Finais}

Na condução clínica do tratamento com pacientes obesos, a percepção de risco da cirurgia bariátrica e das comorbidades associadas à obesidade modifica após as palestras educativas. As intervenções educativas expandem a percepção de risco da cirurgia, mas é independente o risco percebido entre as diferentes comorbidades dos pacientes obesos. Deve ser ressaltado que a dislipidemia é um importante fator de risco para a doença de obesidade e sua compreensão implica mudança de comportamento. Espera-se que esse estudo contribua para ampliar conhecimento sobre o construto da percepção de risco na obesidade, fornecendo subsídios para a qualificação de abordagens terapêuticas multidisciplinares.

\section{Referências}

Adams, T. D., Gress, R. E., Smith, S. C., Halverson, R. C., Simper, S. C., Rosamond, W. D., ...Hunt, S. C. (2007). Long-term mortality after gastric bypass surgery. New England Journal of Medicine, 357(8), 753-761. doi:10.1056/NEJMoa066603

Alvarez, A., Singh, P. M., \& Sinha, A. C. (2014). Postoperative analgesia in morbid obesity. Obesity Surgery, 24(4), 652-659. doi:10.1007/ s11695-014-1185-2

Bonsaksen, T., Lerdal, A., \& Fagermoen, M. S. (2015). Trajectories of illness perceptions in persons with chronic illness: An explorative longitudinal study. Journal of Health Psychology, 20(7), 942-953. doi:10.1177/1359105313504235

Breland, J. Y., Fox, A. M., Horowitz, C. R., \& Leventhal, H. (2012). Applying a common-sense approach to fighting obesity. Journal of Obesity, 2012, 710427. doi:10.1155/2012/710427

Brzozowska, M. M., Sainsbury, A., Eisman, J. A., Baldock, P. A., \& Center, J. R. (2013). Bariatric surgery, bone loss, obesity and possible mechanisms. Obesity Reviews, 14(1), 52-67. doi:10.1111/j.1467-789X.2012.01050.X

Chang, Y., Kim, B. K., Yun, K. E., Cho, J., Zhang, Y., Rampal, S., ...Ryu, S. (2014). Metabolically-healthy obesity and coronary artery calcification. Journal of the American College of Cardiology, 63(24), 2679-2686. doi:10.1016/j. jacc.2014.03.042

Cohen, R., Schiavon, C. A., \& Torres, M. C. (2010). Cirurgia metabólica: Mudanças na anatomia gastrointestinal e a remissão do diabetes mellitus tipo 2. ABCD. Arquivos Brasileiros de $\mathrm{Ci}$ rurgia Digestiva, 23(1), 40-45. doi:http://dx.doi. org/10.1590/S0102-67202010000100010

Flum, D. R, Belle, S. H., Berk, P., Chapman, W., Courcoulas, A., King, W. C., ...Yanovski, S. Z. (2009). Perioperative safety in the longitudinal assessment of bariatric surgery. New England Journal of Medicine, 361(5), 445-454. doi:10.1056/NEJMoa0901836

Friedman, K. E., Applegate, K. L., \& Grant, J. (2007). Who is adherent with preoperative psychological treatment recommendations among weight loss surgery candidates? Surgery for Obesity and Related Diseases, 3(3), 376-382. doi:http:// dx.doi.org/10.1016/j.soard.2007.01.008

Holtorf, A. P., Rinde, H., Rupprecht, F., Alder, H., $\&$ Brixner, D. (2012). The economic impact of bariatric surgery. Advanced Bariatric and Metabolic Surgery. doi:10.5772/33003

Jumean, M. F., Korenfeld, Y., Somers, V. K., Vickers, K. S., Thomas, R. J., \& Lopez-Jimenez, F. (2012). Impact of diagnosing metabolic syndrome on risk perception. American Journal of Health Behavior, 36(4), 522-532. doi:10.5993/ AJHB.36.4.9

Kaly, P., Orellana, S., Torrella, T., Takagishi, C., Saff-Koche, L., \& Murr, M. M. (2008). Unrealistic weight loss expectations in candidates for bariatric surgery. Surgery for Obesity and Related Diseases, 4(1), 6-10. doi:10.1016/j.soard.2007.10.012 
Kubik, J. F., Gill, R. S., Laffin, M., \& Karmali, S. (2013). The impact of bariatric surgery on psychological health. Journal of Obesity, 2013, 837989. doi:10.1155/2013/837989

Lee, C. J., Clark, J. M., Asamoah, V., Schweitzer, M., Magnuson, T., \& Lazo, M. (2015). Prevalence and characteristics of individuals without diabetes and hypertension who underwent bariatric surgery: Lessons learned about metabolically healthy obese. Surgery for Obesity and Related Diseases, 11, 142-147. doi:10.1016/j. soard.2014.06.012

Murguía, M. S., Sierra, A. V., Barragán, V. R, Hernandéz, J. C. L., Rodriguéz, M. E. R., \& Tamayo, M. T. (2012). Psychosocial profile of bariatric surgery candidates and the correlation between obesity level and psychological variables. International Journal of Psychology \& Psychological Therapy, 12(3), 405-414.

Pona, A. A., Heinberg, L. J., Lavery, M., Ben-Porath, Y. S., \& Rish, J. M. (2016). Psychological predictors of body image concerns 3 months after bariatric surgery. Surgery for Obesity and Related Diseases, 12(1), 188-193. doi:10.1016/j. soard.2015.05.008

Prasad, C., Batsis, J. A., Lopez-Jimenez, F., Clark, M. M., Somers, V. K., Sarr, M. G., \& Collazo-Clavell, M. L. (2014). Risk perception of obesity and bariatric surgery in patients seeking treatment for obesity. European Journal of Preventive Cardiology, 21(6), 692-703. doi:10.1177/2047487312466904

Ribeiro, G. A. N. de A., Santos, J. E. dos, \& Loureiro, S. R. (2011). Perfil psicológico de mulheres e a cirurgia bariátrica: Estudo exploratório. Revista Interamericana de Psicologia, 2(45), 169-176.

Schauer, D. P., Arterburn, D. E., Wise, R., Boone, W., Fischer, D., \& Eckman, M. H. (2013). Predictors of bariatric surgery among an interested population. Surgery for Obesity and Related Diseases. doi:10.1016/j.soard.2013.09.014

Shiloh, S., Wade, C. H., Roberts, J. S., Alford, S. H., \& Biesecker, B. B. (2013). Associations between risk perceptions and worry about common diseases: A between- and within-subjects examination. Psychology \& Health, 28(4), 434-449. doi:10.1080/08870446.2012.737464
Sjöberg, L. (1998). Worry and risk perception. Risk Analysis, 18(1), 85-93. doi:10.1111/j. 15396924.1998.tb00918.x

Sjöström, L., Lindroos, A. K., Peltonen, M., Torgerson, J., Bouchard, C., Carlsson, B., ...Swedish Obese Subjects Study Scientific Group. (2004). Lifestyle, diabetes, and cardiovascular risk factors 10 years after bariatric surgery. New England Journal of Medicine, 351(26), 2683-2693. doi:10.1056/NEJMoa035622

Sjöström, L., Narbro, K., Sjöström, C. D., Karason, K., Larsson, B., Wedel, H., ...Swedish Obese Subjects Study. (2007). Effects of bariatric surgery on mortality in Swedish obese subjects. New England Journal of Medicine, 357(8), 741752. doi:10.1056/NEJMoa066254

Slovic, P., Finucane, M. L., Peters, E., \& MacGregor, D. G. (2004). Risk as analysis and risk as feelings: Some thoughts about affect, reason, risk, and rationality. Risk Analysis, 24(2), 311-322. doi:10.1111/j.0272-4332.2004.00433.x

Steffen, K. J., Sarwer, D. B., Thompson, J. K., Mueller, A., Baker, A. W., \& Mitchell, J. E. (2012). Predictors of satisfaction with excess skin and desire for body contouring after bariatric surgery. Surgery for Obesity and Related Diseases, 8(1), 92-97. doi:10.1016/j.soard.2011.06.022

Wee, C. C., Hamel, M. B., Apovian, C. M., Blackburn, G. L., Bolcic-Jankovic, D., Colten, M. E., ...Jones, D. B. (2013). Expectations for weight loss and willingness to accept risk among patients seeking weight loss surgery. JAMA Surgery, 148(3), 264-271. doi:10.1001/jamasurg.2013.1048

Wrzesinski, A., Corrêa, J. M., Fernandes, T. M. B., Monteiro, L. F., Trevisol, F. S., \& Nascimento, R. R. (2015). Complications requiring hospital management after bariatric surgery. $A B C D$. Arquivos Brasileiros de Cirurgia Digestiva, 28(Supl. 1), 3-6 doi:http://dx.doi.org/10.1590/ S0102-6720201500S100003

Recebido: 02/10/2016

$1^{a}$ revisão: $30 / 12 / 2016$

$2^{a}$ revisão: $15 / 02 / 2017$

Aceite final: 16/02/2017

(C) O(s) autor(es), 2018. Acesso aberto. Este artigo está distribuído nos termos da Licença Internacional Creative Commons Atribuição 4.0 (http://creativecommons.org/licenses/by/4.0/), que permite o uso, distribuição e reprodução sem restrições em qualquer meio, desde que você dê crédito apropriado ao(s) autor(es) original(ais) e à fonte, fornecer um link para a licença Creative Commons e indicar se as alterações foram feitas. 